\title{
Recurrence rate and progression of chondrosarcoma is correlated with heat shock protein expression
}

\author{
KLEMENS TRIEB $^{1}$, IRENE SULZBACHER ${ }^{2}$ and BERND KUBISTA ${ }^{3}$ \\ ${ }^{1}$ Department of Orthopaedics, Hospital Wels-Grieskirchen, Wels A-4600; Departments of ${ }^{2}$ Pathology and ${ }^{3}$ Orthopaedics, \\ University Hospital of Vienna, Vienna A-1090, Austria
}

Received November 10, 2014; Accepted September 4, 2015

DOI: $10.3892 / \mathrm{ol} .2015 .3926$

\begin{abstract}
Heat shock proteins (HSPs) are involved in tumour immunity, and are correlated with survival and drug resistance in numerous types of cancer. The present study investigated the expression of HSPs and multiple drug resistance (MDR) in human chondrosarcoma. HSP and P-glycoprotein (the MDR1 gene product) expression was evaluated by immunohistochemical analysis of paraffin-embedded sections obtained from 37 patients with chondrosarcoma (19 male and 18 female; aged 33-85 years; mean age, 48.5 years). HSP73 and 90 were significantly overexpressed in patients with local recurrence: HSP73 was expressed in 7/7 patients $(100 \%)$ with local recurrence and $9 / 18$ patients $(50 \%)$ without recurrence $(\mathrm{P}<0.02)$, while HSP90 was expressed in all patients with recurrence but only 8/18 $(44 \%)$ without recurrence $(\mathrm{P}<0.02)$. A marked association was also identified between HSP expression and survival. HSP72 and 73 were significantly overexpressed in tumours from patients who succumbed to the disease (all positive for HSP72 and 73; P<0.05). No differences were observed between HSP27, 73 or 90-positive or -negative tumours according to age or gender. In addition, HSP72 expression was correlated with differentiation of the tumours $(\mathrm{P}<0.02)$. These results indicate that HSP72, 73 and 90 may function as novel prognostic markers for chondrosarcoma, and initiate further studies regarding the use of such markers for the identification of patients with poor prognosis.
\end{abstract}

\section{Introduction}

Chondrosarcoma is the third most common malignant bone tumour, following osteosarcoma and Ewing's sarcoma (1).

Correspondence to: Professor Klemens Trieb, Department of Orthopaedics, Hospital Wels-Grieskirchen, 42 Grieskirchnerstrasse, Wels A-4600, Austria

E-mail: klemens.trieb@klinikum-wegr.at

Abbreviations: HSPs, heat shock proteins

Key words: chondrosarcoma, heat shock proteins, prognostic marker, differentiation
Chondrosarcoma most often occurs in the pelvis, and exhibits a peak manifestation during the fifth and seventh decades (1).

Chondrosarcoma is unresponsive to chemotherapy or radiotherapy, and until now surgery has remained the only available effective treatment for chondrosarcoma. Resection with wide margins is the treatment of choice for all chondrosarcomas, following the confirmation of diagnosis with a biopsy (2). There are several known clinical diagnostic or prognostic factors for chondrosarcoma, including tumour size, location and histological grading $(1,2)$, however these remain poorly-defined (3-7). In osteosarcoma, a novel group of markers belonging to the heat shock protein (HSP) family has been established $(7,8)$. It was demonstrated that HSP72 de novo expression correlated with response to neoadjuvant chemotherapy; HSP72-positive osteosarcomas exhibited improved responses to chemotherapy compared with those of HSP72-negative cases (8). A previous study revealed a correlation between HSP72 expression and tumor differentiation (9). HSPs are highly conserved and physiologically essential proteins, and numerous types of HSP have been identified to date (10-12).

Under normal conditions HSPs function as molecular chaperones, preventing incorrect interactions and assisting in the assembly of other proteins in various cell compartments. In the case of harmful events, certain HSPs are upregulated to increase cellular resistance to injury and prevent cell death (13).

HSP expression in malignant tumours differs to that of normal tissues, and may be correlated with poor prognosis (12). HSPs also have a role in immunology (14-16), and are involved in the processing and presentation of antigens, as well as the protection of tumour cells from lysis by tumour necrosis factor (17). HSP72 is selectively expressed on the surface of tumour cells, where it elicits an antitumour response, mediated by natural killer cells (16). A correlation between HSP27 expression and tumourigenicity has also been demonstrated (18).

Drug resistance remains one of the major limitations of cancer treatment (19-23). P-glycoprotein, the protein product of the multiple drug resistance (MDR) 1 gene is hypothesised to function as an adenosine triphosphate-dependent efflux pump, responsible for the active removal of chemotherapeutic agents from tumour cells $(22,23)$. In order to evaluate the prognostic value of MDR proteins in chondrosarcoma, the expression of this type of protein was investigated.

The purpose of the present study was to investigate the expression of HSPs and P-glycoprotein (MDR1) in 
Table I. Characteristics and treatment of patients with chondrosarcoma $(\mathrm{n}=37)$.

\begin{tabular}{lc}
\hline Characteristic & Patients, $\mathrm{n}(\%)$ \\
\hline Gender & \\
Male & $19(51.4)$ \\
Female & $18(48.6)$ \\
Mean age, years & \\
Females & 50.4 \\
Males & 46.7 \\
Localisation & \\
Pelvis & $15(40.6)$ \\
Femur & $5(13.5)$ \\
Tibia & $5(13.5)$ \\
Humerus & $5(13.5)$ \\
Scapula & $2(5.4)$ \\
Costa & $1(2.7)$ \\
Metacarpus & $2(5.4)$ \\
Acetabulum & $1(2.7)$ \\
Sacrum & $1(2.7)$ \\
Surgery (n=35) & \\
Endoprosthesis & $16(45.7)$ \\
Amputation & $10(28.6)$ \\
Resection & $9(25.7)$ \\
\hline
\end{tabular}

chondrosarcoma by immunohistochemistry, and to investigate their potential correlation with certain clinical parameters.

\section{Patients and methods}

Patients. A total of 37 patients with chondrosarcoma (19 males and 18 females; aged 33-85 years; mean age, 48.5 years), recruited from University Hospital of Vienna (Vienna, Austria) between May 2001 and November 2004, were analysed in the present study. Tumour biopsies were obtained prior to any treatment. The pelvis was the most frequent tumour location ( 15 cases), followed by the femur, tibia and humerus (5 cases each), scapula ( 2 cases), metacarpus ( 2 cases), costa, acetabulum and sacrum (1 case each).

In total, 35 patients were treated with surgery; one patient died prior to surgery, immediately following biopsy, and in one case the location (sacrum) made surgery impossible. Amputations were required in 10 cases, and resection in 9 cases (Table I). Histopathological grading of the biopsy samples was as follows: G1, 3 patients; G2, 16 patients; and G3, 18 cases. Local recurrence was identified in 7 patients.

Patients were clinically followed up for a minimum of 24 months after surgery, with a mean \pm standard deviation follow-up time of 5.9 \pm 0.7 years (range, 2.0-8.2 years). Written informed consent was received from the patients or the patients' family.

Immunohistochemistry. Biopsy specimens (2-3 $\mu \mathrm{m}$ thick) were frozen to $-80^{\circ} \mathrm{C}$ using liquid nitrogen, sectioned, fixed in
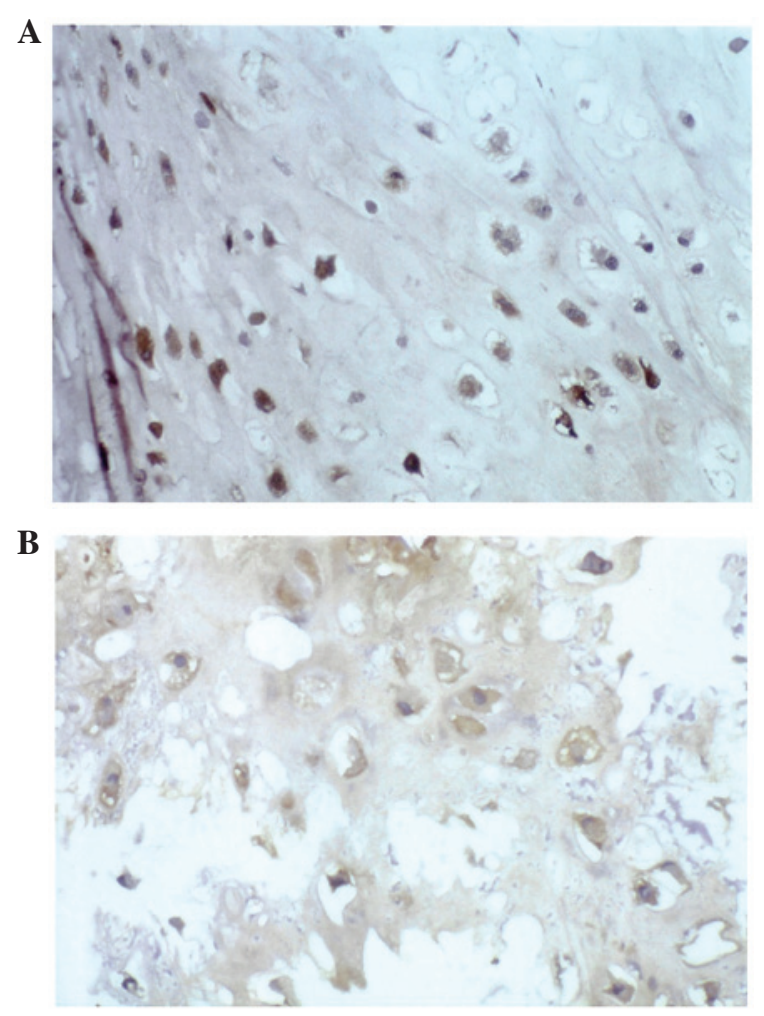

Figure 1. HSPs and chondrosarcoma. (A) Immunostaining for HSP60 revealed exclusive cytoplasmic expression of this protein (immunoperoxidase stain; original magnification, x40). (B) Immunostaining for HSP72 indicated strong and diffuse expression of HSP72 (immunoperoxidase stain; original magnification, $\mathrm{x} 40)$.HSPs, heat shock proteins.

7.5\% formalin and embedded in paraffin (Sigma-Aldrich, St. Louis, MO, USA). Protease treatment (type XIV, Sigma P5147; Sigma-Aldrich) of the sections was used for antigen retrieval. Following deparaffinisation and rehydration of slides, immunohistochemistry was performed using specific monoclonal mouse anti-human antibodies against HSP27 (1:200 dilution; cat no. SPA802), HSP60 (1:250 dilution; cat no. SPA804), HSP72 (1:200 dilution; cat no. SPA810), HSP73 (1:300 dilution; cat no. SPA815) and HSP90 (1:250 dilution; cat no. SPA817; Stressgene, Victoria, Canada), as well as polyclonal goat anti-human MDR (1:200 dilution; cat no. sc-1517; Santa Cruz Biotechnology, Inc., Dallas, TX, USA) for $1 \mathrm{~h}$ at room temperature. Biotinylated horse anti-mouse or anti-goat IgG secondary antibodies (1:100 dilution; cat. no. BA-1300) were applied for $30 \mathrm{~min}$ at room temperature, followed by the application of Streptavidin Biotin complex (ABC Vectastain Elite PK-6100; Vector Laboratories, Inc., Burlingame, CA, USA) together with diaminobenzidine-development (Sigma-Aldrich Switzerland, Buchs, Switzerland) for $30 \mathrm{~min}$ at room temperature for visualisation of the immune reaction. Immunostained sections were semiquantitatively evaluated by light microscopy (Axio Examiner; Carl Zeiss GmbH, Jena, Germany) by two observers and scored positive when $>10 \%$ of tumour cells were stained.

Statistics. Statistical evaluation was performed on an Apple Macintosh computer using StatView II software (version 2.0; SAS Institute Inc., Cary, NC, USA). Continuous data and ordered categories were compared using the Mann-Whitney U test 
A
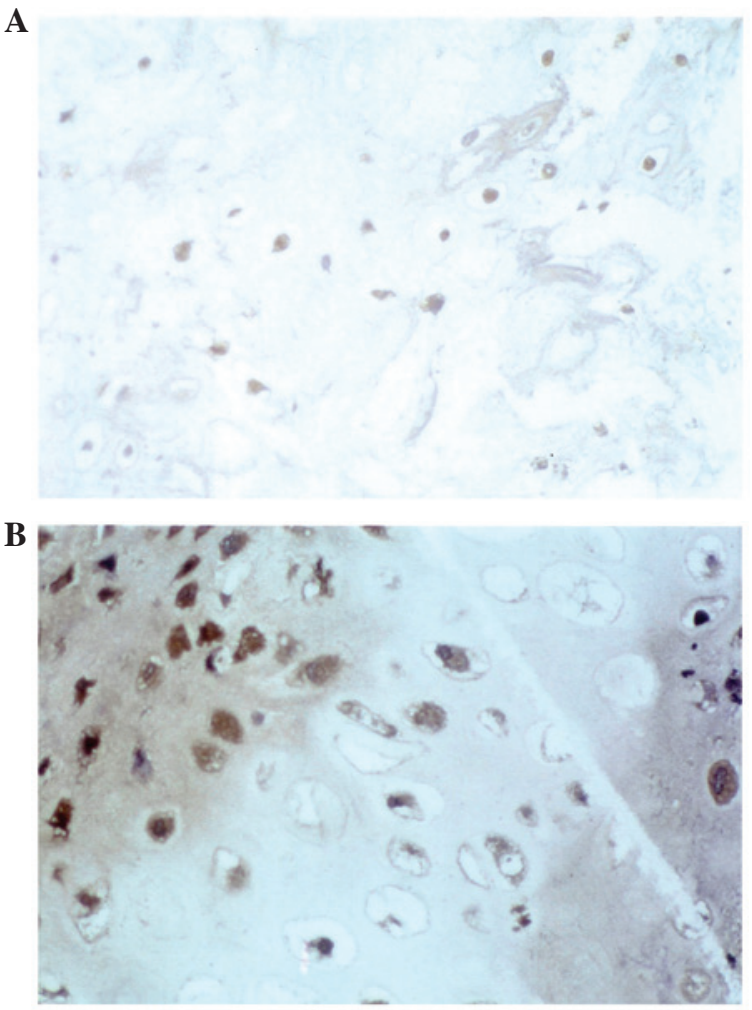

Figure 2. HSPs and chondrosarcoma. (A) Immunostaining for HSP73 revealed exclusive cytoplasmic expression of this protein (immunoperoxidase stain original magnification, x40). (B) Immunostaining for HSP90 (immunoperoxidase stain; original magnification, x60) HSPs, heat shock proteins.

corrected for ties (other contingency tables were analysed for differences using the $\chi^{2}$ test). The chondrosarcoma specimens were divided in 2 groups for analysis, according to the expression of each protein: Expressing and non-expressing.

\section{Results}

HSP expression. In the biopsy specimens from patients with chondrosarcoma $(n=37), 23 / 37$ cases $(62 \%)$ were positive for HSP27 expression (data not shown), 22/37 (59\%) were positive for HSP60 expression (Fig. 1A), 19/37 (51\%) expressed HSP72 (Fig. 1B), HSP73 was expressed in 23/37 (62\%) (Fig. 2A), 23/37 (62\%) were positive for HSP90 expression (Fig. 2B) and MDR expression was positive in 18/37 cases (49\%; data not shown).

HSP72 and 90 are associated with local recurrence. A marked correlation was detected between HSP72 and 90 expression and local recurrence $(\mathrm{P}<0.02$; Table II). HSP73 was expressed in $7 / 7$ cases $(100 \%)$ positive for local recurrence, compared with only $9 / 18$ cases $(50 \%)$ exhibiting no recurrence $(\mathrm{P}<0.02)$. For HSP90, 7/7 cases $(100 \%)$ with local recurrence were positive, while $8 / 18$ cases $(44 \%)$ without recurrence were positive $(\mathrm{P}<0.02)$. No such correlation was identified between HSP27, HSP60, HSP73 or MDR expression, and local recurrence, as shown in Table II (patients with a follow-up period of $<30$ months were excluded).

HSP72 and 73 are prognostic factors. All the patients included in the present study who succumbed to the disease
Table II. Recurrence rate of patients with chondrosarcoma $(n=25)$.

Protein expression Recurrence, $\mathrm{n}(\%)$ No recurrence, $\mathrm{n}(\%)$

$\begin{array}{llr}\text { HSP27 } & & \\ \text { Positive } & 4(57) & 7(39) \\ \text { Negative } & 3(43) & 11(61) \\ \text { HSP60 } & & 8(44) \\ \text { Positive } & 6(86) & 10(56) \\ \text { Negative } & 1(14) & \\ \text { HSP72 } & & 7(39) \\ \text { Positive } & 6(86)^{\mathrm{a}} & 11(61) \\ \text { Negative } & 1(14) & 9(50) \\ \text { HSP73 } & & 9(50) \\ \text { Positive } & 7(100) & 8(44) \\ \text { Negative } & 0(0) & 10(56) \\ \text { HSP90 } & & 8(44) \\ \text { Positive } & 7(100)^{\mathrm{a}} & 10(56) \\ \text { Negative } & 0(0) & \\ \text { MDR } & & \\ \text { Positive } & 4(57) & \\ \text { Negative } & 3(43) & \end{array}$

${ }^{\mathrm{a}} \mathrm{P}<0.05$; HSP, heat shock protein; MDR, multiple drug resistance.

Table III. DOD rate of patients with chondrosarcoma $(n=28)$.

\begin{tabular}{|c|c|c|}
\hline \multirow[b]{2}{*}{ Protein expression } & \multicolumn{2}{|c|}{ DOD, n (\%) } \\
\hline & Yes & No, $n$ \\
\hline \multicolumn{3}{|l|}{ HSP27 } \\
\hline Positive & $3(43)$ & $9(43)$ \\
\hline Negative & $4(57)$ & $12(57)$ \\
\hline \multicolumn{3}{|l|}{ HSP60 } \\
\hline Positive & $5(71)$ & $11(52)$ \\
\hline Negative & $2(29)$ & $10(48)$ \\
\hline \multicolumn{3}{|l|}{ HSP72 } \\
\hline Positive & $7(100)^{\mathrm{a}}$ & $9(43)$ \\
\hline Negative & $0(0)$ & $12(57)$ \\
\hline \multicolumn{3}{|l|}{ HSP73 } \\
\hline Positive & $7(100)^{\mathrm{a}}$ & $9(43)$ \\
\hline Negative & $0(0)$ & $12(57)$ \\
\hline \multicolumn{3}{|l|}{ HSP90 } \\
\hline Positive & $6(86)$ & $11(52)$ \\
\hline Negative & $1(14)$ & $10(48)$ \\
\hline \multicolumn{3}{|l|}{ MDR } \\
\hline Positive & $6(86)$ & $11(52)$ \\
\hline Negative & $1(14)$ & $10(48)$ \\
\hline
\end{tabular}

${ }^{\mathrm{a}} \mathrm{P}<0.05$; HSP, heat shock protein; MDR, multiple drug resistance; DOD, dead of disease. 
were HSP72- and 73-positive. For HSP72, 7/7 patients (100\%) dead of disease (DOD) were HSP72 positive, while 9/21 patients $(43 \%)$ not DOD were HSP72 positive $(\mathrm{P}<0.009)$. For HSP73, 7/7 patients (100\%) DOD were HSP73 positive, whereas 9/21 patients (43\%) not DOD were HSP73 positive $(\mathrm{P}<0.009)$. There was thus a marked correlation between HSP72 and 73 expression and the DOD rate $(\mathrm{P}<0.009)$, shown in Table III (patients with a follow-up period $<36$ months were excluded).

HSP72 and 73 are correlated with tumour differentiation. Concurrently with the results of a previous study, HSP72 expression in chondrosarcoma was demonstrated to correlate with tumour differentiation $(\mathrm{P}<0.019)$ : 3/3 G1 cases $(100 \%), 10 / 16 \mathrm{G} 2$ (63\%) and 6/18 G3 (33\%) were HSP72 positive (9). A correlation was also revealed between HSP73 and differentiation, although this was less marked $(\mathrm{P}<0.05)$. No such correlation was observed for HSP27, 60, 90 or MDR (data not shown).

\section{Discussion}

To date, few studies exist regarding the expression of HSP in mesenchymal tissue. A controversial role for HSP27 in differentiation and drug resistance has previously been described (18). The induction of HSP27 expression in HSP27-negative chondrosarcomas may result in enhanced lysis of tumour cells by lymphocytes and therefore may represent a novel target for therapeutic approaches $(10,11)$.

A marked correlation between HSP72 and 73 expression, and the DOD rate was observed. All patients who succumbed chondrosarcoma were HSP72 and 73 positive, which may support the use of HSP72 and 73 as significant prognostic markers in chondrosarcoma.

Notably, a marked correlation between HSP72 and 90 expression, and the appearance of local recurrence was identified. Therefore the role of HSP72, 73 and 90 is likely to be significant in chondrosarcoma.

Conversely, the decreased expression of HSP72 in chondrosarcoma was correlated with a lower differentiation status of the tumour. The present study also identified a significant correlation between HSP73 expression and differentiation, however no such correlation was detected for HSP27, 60, 90 or MDR.

The decreased expression of HSP in poorly differentiated chondrosarcomas may explain their highly aggressive behaviour, which may be due to their impaired recognition by the immune system (14). The HSP70 family, and potentially other HSPs, may contribute to reducing tolerance to otherwise hidden tumour antigens by making them recognisable to lymphocytes.

The decreased HSP72 and 73 expression observed in chondrosarcomas, in addition to their correlation with differentiation, may explain why chondrosarcomas are unresponsive to chemotherapy. As chondrosarcomas do not respond to chemotherapy or radiation, wide resection is the therapy of choice. Induction of HSPs presents a novel therapeutic approach for the treatment of chondrosarcomas. HSP induction may be achieved by inducing hyperthermia, as HSPs are stress and heat inducible. Alternatively, a more sophisticated method of induction would be via microwave application.
Therefore, HSPs may be useful in the development of novel therapeutic strategies for chondrosarcoma, and the subsequent improvement of clinical outcomes.

\section{References}

1. Sheth DS, Yasko AW, Johnson ME, Ayala AG, Murray JA and Romsdahl MM: Chondrosarcoma of the pelvis. Prognostic factors for 67 patients treated with definitive surgery. Cancer 78: 745-750, 1996.

2. Evans HL, Ayala AG and Romsdahl MM: Prognostic factors in chondrosarcoma of bone: A clinicopathologic analysis with emphasis on histologic grading. Cancer 40: 818-831, 1977.

3. Kreicbergs A, Boquist L, Borssén B and Larsson SE: Prognostic factors in chondrosarcoma: A comparative study of cellular DNA content and clinicopathologic features. Cancer 50: 577-583, 1982.

4. Springfield DS, Gebhardt MC and McGuire MH: Chondrosarcoma: A review. Instr Course Lect 45: 417-424, 1996.

5. Ayala AG, Ro JY, Han W, Sahin A and Raymond AK: Chondrosarcoma: A clinocopathologic study of 173 cases with a minimal 5 years follow-up. Lab Invest 64: 2A, 1991.

6. Shin KH, Rougraff BT and Simon MA: Oncologic outcomes of primary bone sarcomas of the pelvis. Clin Orthop Relat Res 304: 207-217, 1994.

7. Trieb K, Gerth R, Holzer G, Grohs JG, Berger P and Kotz R: Antibodies to heat shock protein 90 in osteosarcoma patients correlate with response to neoadjuvant chemotherapy. Br J Cancer 82: 85-87, 2000.

8. Trieb K, Lechleitner T, Lang S, Windhager R, Kotz R and Dirnhofer S: Heat shock protein 72 expression in osteosarcomas correlates with good response to neoadjuvant chemotherapy. Hum Pathol 29: 1050-1055, 1998.

9. Trieb K, Kohlbeck R, Lang S, Klinger H, Blahovec H and Kotz R: Heat shock protein 72 expression in chondrosarcoma correlates with differentiation. J Cancer Res Clin Oncol 126: 667-670, 2000.

10. Welch WJ: Mammalian stress response: Cell physiology, structure/function of stress proteins, and implications for medicine and disease. Physiol Rev 72: 1063-1081, 1992.

11. Jäättelä $M$ and Wissing D: Emerging role of heat shock proteins in biology and medicine. Ann Med 24: 249-258, 1992.

12. Lindquist S and Craig EA: The heat-shock proteins. Annu Rev Genet 22: 631-677, 1988.

13. Arrigo AP and Landry J: Expression and function of the low-molecular-weight heat shock proteins. In: The Biology of Heat Shock Proteins and Molecular Chaperones. Morimoto R, Tissieres A and Georgopoulos C (eds). Cold Spring Harbor Press, New York, NY, pp335-373, 1994.

14. Kaufmann SH: Heat shock proteins and the immune response. Immunol Today 11: 129-136, 1990.

15. Udono $\mathrm{H}$ and Srivastava PK: Heat shock protein 70 -associated peptides elicit specific cancer immunity. J Exp Med 178: 1391-1396, 1993.

16. Multhoff G, Botzler C, Jennen L, Schmidt J, Ellwart J and Issels R: Heat shock protein 72 on tumor cells: A recognition structure for natural killer cells. J Immunol 158: 4341-4350, 1997.

17. Gromkowski SH, Yagi J and Janeway CA Jr: Elevated temperature regulates tumor necrosis factor-mediated immune killing. Eur J Immunol 19: 1709-1714, 1989.

18. Guénal I, Sidoti-de Fraisse C, Gaumer S and Mignotte B: Bcl-2 and $\mathrm{Hsp} 27$ act at different levels to suppress programmed cell death. Oncogene 15: 347-360, 1997.

19. Conroy SE and Latchman DS: Do heat shock proteins have a role in breast cancer? Br J Cancer 74: 717-721, 1996.

20. Wunder JS, Bull SB, Aneliunas V, et al: MDR1 gene expression and outcome in osteosarcoma: A prospective, multicenter study. J Clin Oncol 18: 2685-2694, 2000.

21. Borst P: Genetic mechanisms of drug resistance. A review. Acta Oncol 30: 87-105, 1991.

22. Gottesman MM and Pastan I: Biochemistry of multidrug resistance mediated by the multidrug transporter. Annu Rev Biochem 62: 385-427, 1993.

23. Endicott JA and Ling V: The biochemistry of P-glycoprotein-mediated multidrug resistance. Annu Rev Biochem 58: 137-171, 1989. 